\title{
Cryptogenic Fibrosing Pleuritis
}

\author{
Alessandra Manco ${ }^{1}$, Veronica Alfieri ${ }^{1}$, Letizia Gnetti ${ }^{2}$, Marina Aiello $^{1}$, Giuseppina Bertorelli $^{1}$, Maria Majori ${ }^{3}$, Alfredo Chetta $^{1}$ \\ ${ }^{1}$ Department of Medicine and Surgery, Respiratory Disease and Lung Function Unit, University of Parma, Parma, Italy \\ 2 Pathology Unit, University Hospital of Parma, Parma, Italy
}

${ }^{3}$ Department of Cardiothoracic and Vascular Diseases, Pneumology and Endothoracic Endoscopy Unit, University Hospital of Parma, Parma, Italy

\section{Doi: 10.12890/2021_002498- European Journal of Case Reports in Internal Medicine - 다 EFIM 2021}

Received: 18/03/2021

Accepted: 20/03/2021

Published: 17/05/2021

How to cite this article: Manco A, Alfieri V, Gnetti L, Aiello M, Bertorelli G, Majori M, Chetta A. Cryptogenic fibrosing pleuritis. EJCRIM 2021;8: doi:10.12890/2021_002498.

Conflicts of Interests: The authors declare there are no competing interests.

This article is licensed under a Commons Attribution Non-Commercial 4.0 License

\section{ABSTRACT}

We report the case of a 46-year-old male patient who was referred for chest pain and bilateral pleural effusion. Despite treatment with antibiotics and steroids, the pleural effusion worsened over a few months until pulmonary function was halved. The CT scan showed bilateral pleural thickening with right basal opacity. Histology revealed extensive fibrotic tissue with focal collections of lymphocytes and giant cells without traces of asbestos bodies. Since no evidence of an infectious, embolic or occupational aetiology was found, this bilateral pleural effusion progressing to diffuse pleural thickening was diagnosed as cryptogenic fibrosing pleuritis, a rare pleural disease.

\section{LEARNING POINTS}

- Bilateral pleural effusion progressing to diffuse pleural thickening was diagnosed as cryptogenic fibrosing pleuritis, a rare pleural disease.

- Cryptogenic fibrosing pleuritis was treated with high-dose corticosteroids.

- The patient showed stable disease at 6-year follow-up.

\section{KEYWORDS}

Cryptogenic fibrosing pleuritis, pleural thickening, pleural effusion

\section{INTRODUCTION}

Pleural effusions mostly regress spontaneously or with treatment, but in some cases pleural fibrosis develops ${ }^{[1]}$. Pleural thickening can be due to different inflammatory processes, including exposure to asbestos, immunological disease, infection, improperly drained haemothorax, medications, malignancy, the after-effects of coronary bypass surgery, or uraemic pleurisy ${ }^{[1]}$. Unilateral pleural thickening is a common radiographic finding, while bilateral pleural fibrotic sequelae are less common ${ }^{[1]}$.

Cryptogenic bilateral fibrosing pleuritis is a rare condition ${ }^{[2-5]}$, which was first described by Buchanan et al ${ }^{[[]}$, and is characterized by bilateral pleural thickening in the absence of an infectious, embolic or occupational aetiology. Patients usually present chest pain, dyspnoea, cough or malaise ${ }^{[2-5]}$.

We report the case of a patient with progressive disabling pleural fibrosis of unknown aetiology. We followed the patient up from 2013 to 2019.

\section{CASE DESCRIPTION}

In October 2012, a 46-year-old male patient complained of bilateral chest pain exacerbated by breathing and lying in the supine position. In November 2012, he went to the emergency room because of chest pain recurrence and chest radiography showed right pleural effusion. He was discharged on a 10-day course of antibiotic (cefepime and ciprofloxacin) and steroid (betamethasone) therapy. In December 2012, 
despite treatment, symptoms worsened and he was admitted to the lung unit of an associated hospital. A chest CT scan confirmed right pleural effusion. Blood and pleural fluid investigations, including microbiological and rheumatology tests, were negative, but spirometry revealed a restrictive pattern. The patient was discharged with a 10-day course of antibiotic (piperacillin/tazobactam and clarithromycin) and steroid (prednisone) therapy.

In February 2013, because of persistent chest pain and the onset of dyspnoea on exertion, the patient was referred to our unit. He denied any history of smoking or exposure to organic powders or chemicals. His medical history included obesity (class 1), anxiety and hypertension treated with a beta-blocker. Routine blood tests only revealed increased cupremia. Exhaustive serology for microbiological agents was negative. Tests for rheumatoid factor, antinuclear factor, DNA binding autoantibodies, and immune complexes were negative; complement levels were normal. Sputum was negative for acid- and alcohol-fast bacilli on direct smear and culture. A chest x-ray showed bilateral pleural thickening with minimal bilateral pleural effusion (Fig. 1). A chest CT scan showed parenchymal basal consolidations, bilaterally associated with pleural effusion and initial signs of pleural thickening (Fig. 1). Spirometry showed a moderately severe restrictive pattern.

The patient underwent bronchoscopy and transthoracic needle aspiration of the right lung opacity and no malignant cells were found. Thoracoscopy with parietal pleural biopsy was also performed, which showed collagenous dense fibrosis with minimal reactive capillary proliferation, mild non-specific lymphocytic inflammation, and absence of asbestos bodies (Fig. 2). An exhaustive pathological, environmental and family history together with the above investigations allowed us to exclude known causes of pleural effusion, such as tuberculosis or other infections, connective tissue diseases, cancer, asbestos-related diseases and pulmonary embolism. The findings were compatible with chronic pachypleuritis.
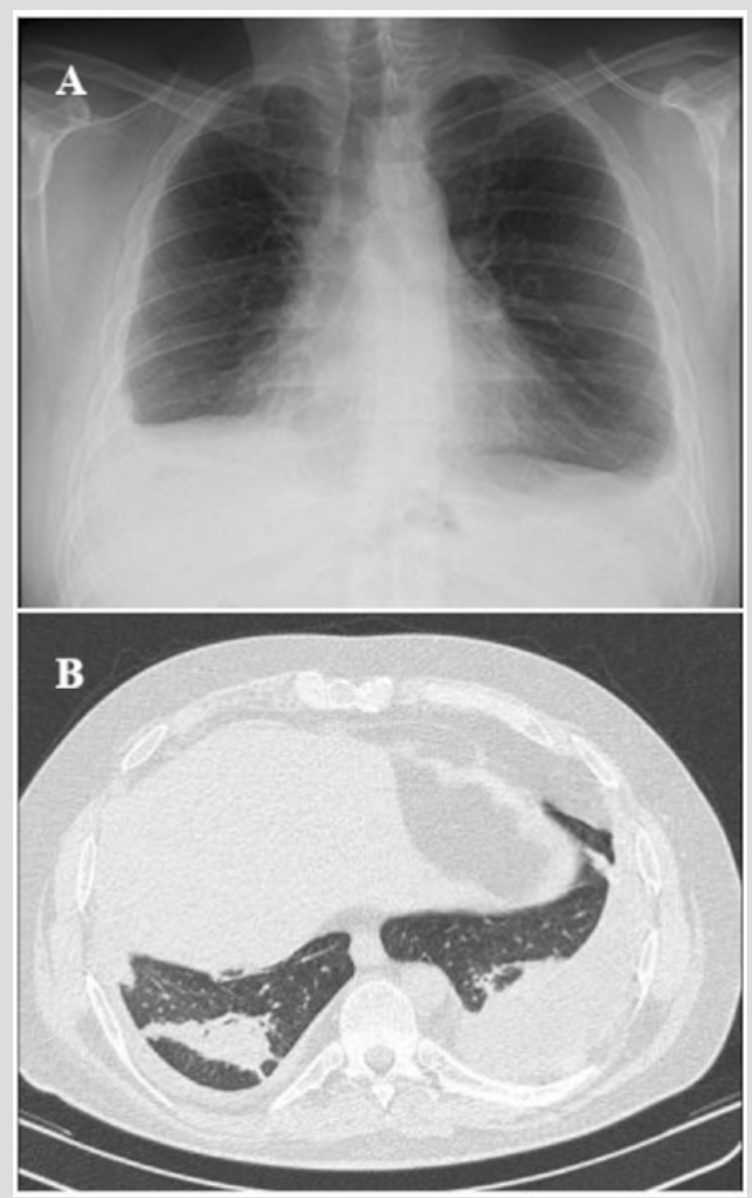

Figure 1. Imaging in 2013. (A) Chest $x$-ray from February 2013 showing bilateral pleural thickening with minimal bilateral pleural effusion, most evident on the right side. (B) Chest CT scan from February 2013 showing the presence of parenchymal basal consolidations, bilaterally associated with pleural effusion with initial signs of bilateral pleural thickening of a maximum thickness of about $20 \mathrm{~mm}$

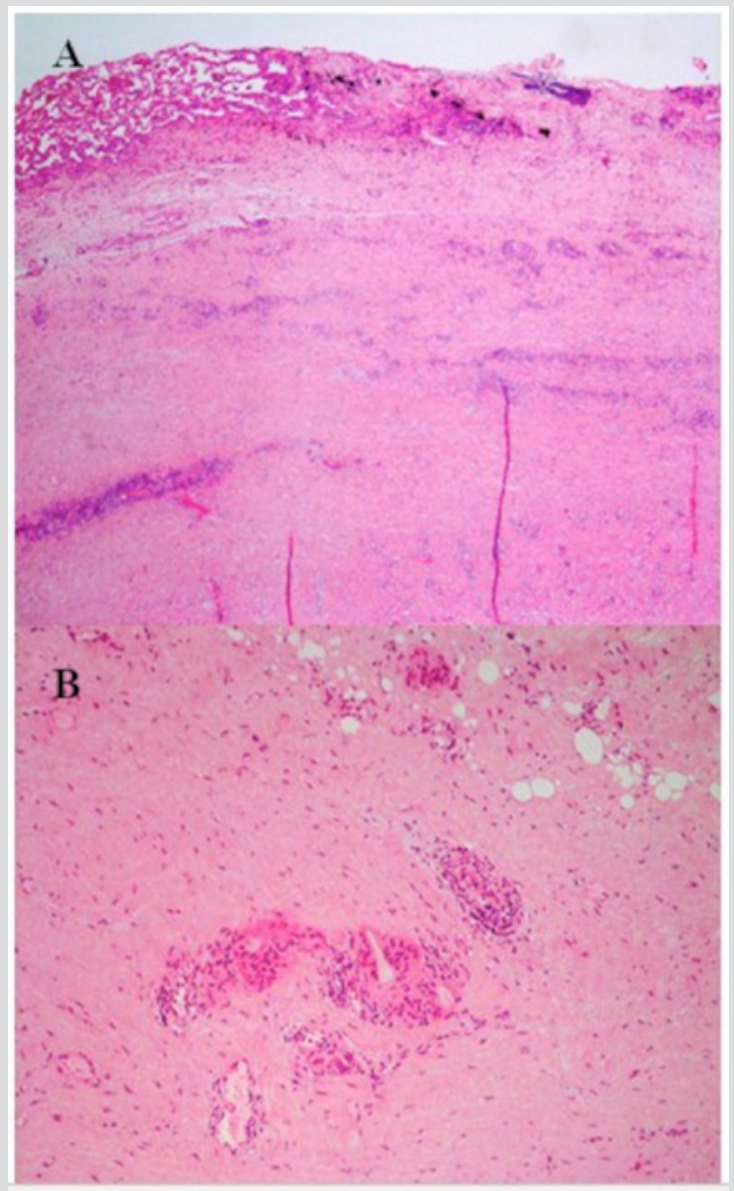

Figure 2. Pleural biopsy histological findings. (A) Extended fibrotic tissue with focal reactive mesothelium. The pleural surface is covered by organizing fibrin. (B) Focal collections of lymphocytes with fibrous tissue intermingled on the subpleural fat. The alveolar spaces are trapped by fibrosis; in some areas foreign-body-type giant cell granulomatous inflammation can be seen 
The case was discussed with thoracic surgeons, radiologists and pathologists and interpreted as cryptogenic fibrosing pleuritis. We administered a high dose of corticosteroids $(0.7 \mathrm{mg} / \mathrm{kg}$ of prednisone for 6 weeks, with tapering over the next 6 months) to the patient. Over the following months, the patient deteriorated functionally (Fig. 3), despite the stabilization of symptoms (chest pain and exertional dyspnoea). However, functional and radiological evaluation has not shown any further worsening since September 2016 (Figs. 3 and 4).

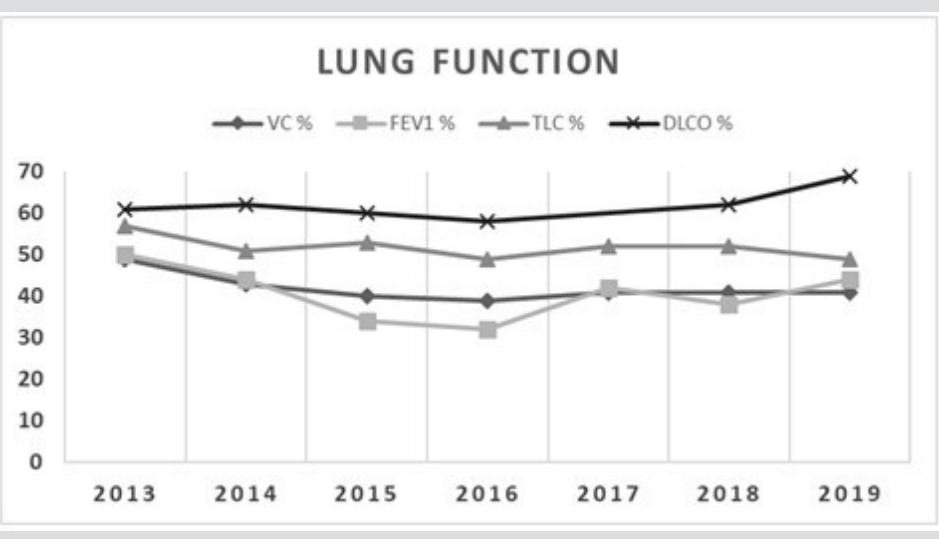

Figure 3. Lung function progression: the graph shows the progressive decline in lung function and the subsequent stability since 2016 up to 2019

Figure 4. Radiological evaluation. (A) Chest x-ray from September 2016 showing a right basal consolidation attributable to a round atelectasis and associated with bilateral pleural effusion. (B) Chest CT scan from July 2018 showing stable radiological findings, confirming the right pleural thickening
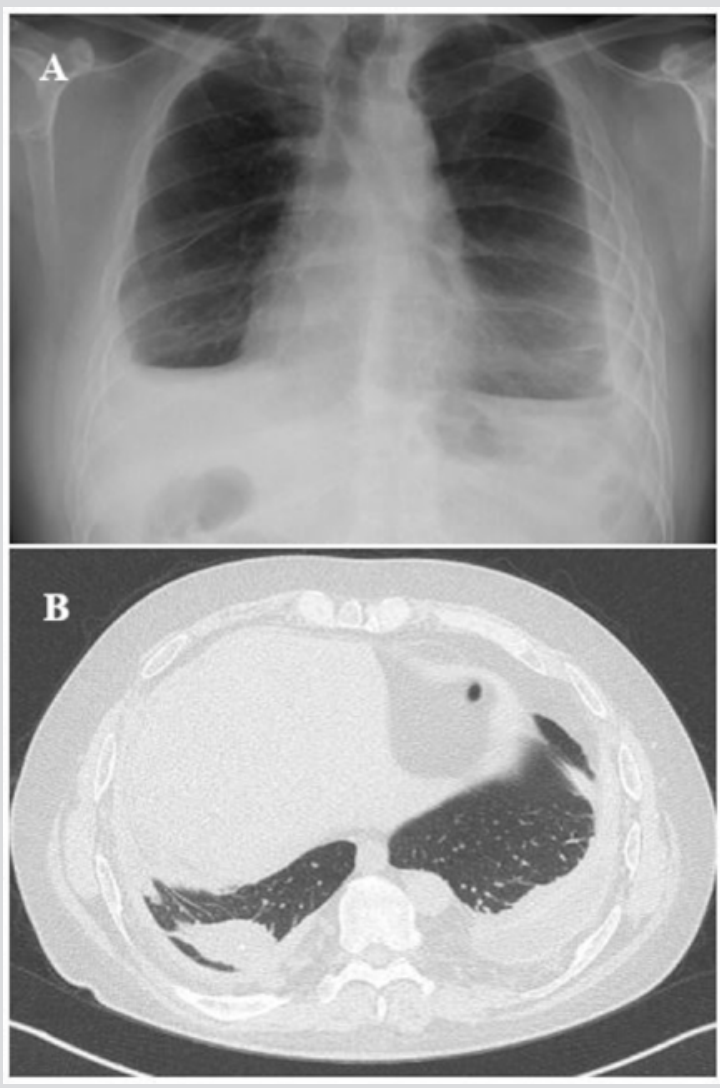

\section{DISCUSSION}

We present a patient with progressive dyspnoea and chest pain with radiological findings highly suggestive of bilateral fibrosing pleuritis. Histology of the pleural biopsy confirmed the presence of extensive fibrotic tissue with reactive mesothelium and focal collections of lymphocytes. A diagnosis of cryptogenic pleurisy requires the exclusion of known causes, including rheumatoid pleurisy, tuberculous pleurisy, fibrosing uremic pleuritis ${ }^{[1]}$, drugs, sarcoidosis, familial polyserositis and recurrent pulmonary embolism ${ }^{[3]}$. Exposure to asbestos was a possibility in our case, but as the patient denied environmental exposure to asbestos and there were no asbestos bodies in the pleural biopsy specimen, we were able to exclude asbestos-related pathologies.

Very few cases of cryptogenic bilateral fibrosing pleuritis have been reported in the literature. The first four were described by Buchanan et al. in $1988^{[3]}$. They showed clinical and radiological manifestations and a restrictive function pattern with low DLCO and normal KCO, comparable to the findings in our patient, who, however, was a non-smoker and HLA-B44 negative. In addition, three of the four cases were treated with pleural decortication and only one with steroid therapy but with fair results in a short evaluation. This supports our therapeutic choice, because after an initial decline, our patient is now stable.

In 1995 Hayes et al. described the case of two brothers with Fanconi's syndrome who developed bilateral fibrosing pleuritis of unknown cause ${ }^{[4]}$, accompanied by respiratory failure. One of these patients died despite therapy with penicillamine, corticosteroids and colchicine. These patients differ from ours in that they also had Fanconi's syndrome (whose correlation with pleural fibrosis is unknown).

Another case described by Lee-Chiong Jr et al. in $1996^{[5]}$ concerned a patient with left pleural thickening, which was treated with pleural decortication. However, 6 months later right pleural fibrosis developed, requiring right pleural decortication. Our patient has asynchronous pleural fibrosis, which is less striking and well controlled with high-dose steroid therapy. 


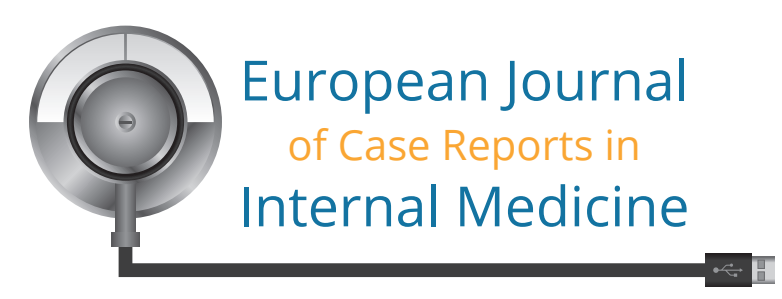

In conclusion, there are very few known cases of cryptogenic fibrosing pleuritis and the therapeutic approach has not yet been properly established. Based on our previous experience, we chose treatment with high-dose steroids to be tapered over a few months. At 6-year follow-up, the patient is functionally and radiologically stable with a consistent quality of life.

Further studies are needed to clarify the pathological mechanisms and evolution of this rare condition and to improve our knowledge of therapeutic possibilities.

\section{REFERENCES}

1. Huggins JT, Sahn SA. Causes and management of pleural fibrosis. Respirology 2004;9:441-447. doi:10.1111/j.1440-1843.2004.00630.x

2. Azoulay E, Paugam B, Heymann MF, Kambouchner M, Haloun A, Valeyre D, et al. Familial extensive idiopathic bilateral pleural fibrosis. Eur Respir J 1999;14:971-973. doi:10.1034/j.1399-3003.1999.14d41.x

3. Buchanan DR, Johnston IDA, Kerr IH, Hetzel MR, Corrin B, Turner-Warwick M. Cryptogenetic bilateral fibrosing pleuritis. Br J Dis Chest 1988;82:186. doi:10.1016/00070971(88)90042-3

4. Hayes JP, Wiggins J, Ward K, Muldowney F, FitzGerald MX. Familial cryptogenic fibrosing pleuritis with Fanconi's syndrome (renal tubular acidosis). A new syndrome. Chest 1995;107:576-578. doi:10.1378/chest.107.2.576

5. Lee-Chiong Jr TL, Hilbert J. Extensive idiopathic benign bilateral asynchronous pleural fibrosis. Chest 1996;109:564-565. doi:10.1378/chest.109.2.564 